\title{
Tuberculosis control has failed in South Africa - time to reappraise strategy
}

\author{
Robin Wood, Stephen D Lawn, Simon Johnstone-Robertson, Linda-Gail Bekker
}

South Africa's rate of tuberculosis (TB) has increased over the last 20 years, to now having the third-highest TB burden in the world. The TB control programme has primarily focused on effective case management of passively presenting TB cases, and progress has been recorded towards international treatment targets. While outcomes for notified TB cases have improved, this strategy failed to contain the TB epidemic. South Africa has the highest per capita annual risk of TB disease of comparably sized countries globally, and its communities have extremely high TB transmission rates. The rates of TB infection of children and adolescents are now similar to those reported 100 years ago in Europe long before chemotherapy became available. High rates of HIV testing of TB patients in Cape Town allows analysis of TB notification data stratified by age, type of TB and HIV status, and a better understanding of TB epidemiology. TB infection prevalence data from Cape Town communities allow estimation of the prevailing force of TB infection and, together with $\mathrm{TB}$ notification and prevalence data, the effective number of secondary infections and case finding proportions can be estimated. This better understanding of the major drivers of the TB epidemic allows reasons to be identified for failure of the present strategy. New control strategies can also be identified, that must be accompanied by novel TB control targets.

S Afr Med J 2010;100:111-114.
South African reports to the World Health Organization (WHO) indicate that its tuberculosis (TB) notifications have increased fivefold over the last 20 years; in 2008, South Africa (SA) had the third-highest TB burden, after India and China. ${ }^{1}$ SA and Swaziland now have the highest TB notification rates in the world, with about $1 \%$ of their populations developing TB annually. ${ }^{1,2} \mathrm{SA}$ was responsible for approximately $25 \%$ of the global burden of HIV-associated TB cases in 2007.

While a worsening epidemic is revealed, data give little insight into understanding why TB control is failing. To better understand the epidemiology of TB control, the national and available community and city level data must be integrated; understanding this provides insights into the weaknesses of existing strategies and permits the development of additional rational interventions to regain $\mathrm{TB}$ control.

Desmond Tutu HIV Centre, and Institute of Infectious Diseases and Molecular Medicine, Faculty of Health Sciences, University of Cape Town

Robin Wood, MB BCh, BSc, FCP

Stephen D Lawn, BMedSci, MB BS, MRCPMD, DTM\&H, Dip HIV Med

Simon Johnstone-Robertson, BSc, BSc (Hons), PDS

Linda-Gail Bekker, MB ChB, FCP, PhD

Department of Medicine, Faculty of Health Sciences, University of Cape Town Robin Wood, MB BCh, BSc, FCP

Linda-Gail Bekker, MB ChB, FCP, PhD

Department of Science and Technology/National Research Foundation, Centre of Excellence in Epidemiological Modeling and Analysis, Stellenbosch University Robin Wood, MB BCh, BSc, FCP

Simon Johnstone-Robertson, BSc, BSc (Hons), PDS

Department of Clinical Research, Faculty of Infectious and Tropical Diseases, London School of Hygiene and Tropical Medicine, London, UK

Stephen D Lawn, MMedSci, MB BS, MRCPMD, DTM\&H, Dip HIV Med

\section{TB notifications}

In 2007, 315000 cases of TB were notified in South Africa - a rate of new and recurrent disease of 649 per 100000 population. $^{2}$ Approximately $40 \%$ of nationally notified cases were tested for HIV infection, of which $73 \%$ were estimated to be positive, but this high prevalence may be affected by selection bias. The 2009 Cape Town (population 3.4 million) notification of $31095 \mathrm{~TB}$ cases $^{3}$ represents double the number of TB cases reported in the USA (population $>300$ million people). ${ }^{2}$

Provider-initiated HIV testing in Cape Town TB clinics has increased to over $85 \%$, allowing analysis of data stratified by age and HIV status, and providing a better understanding of the epidemiology of TB in a large urban population. Using denominators from the Cape Town population structure (Calle Hedberg, City of Cape Town and Stats SA) and TB notification data, age-specific TB notification rates stratified by HIV status were derived (Fig. 1). HIV-associated TB accounted for $44 \%$ of the total case burden. The peak TB notification

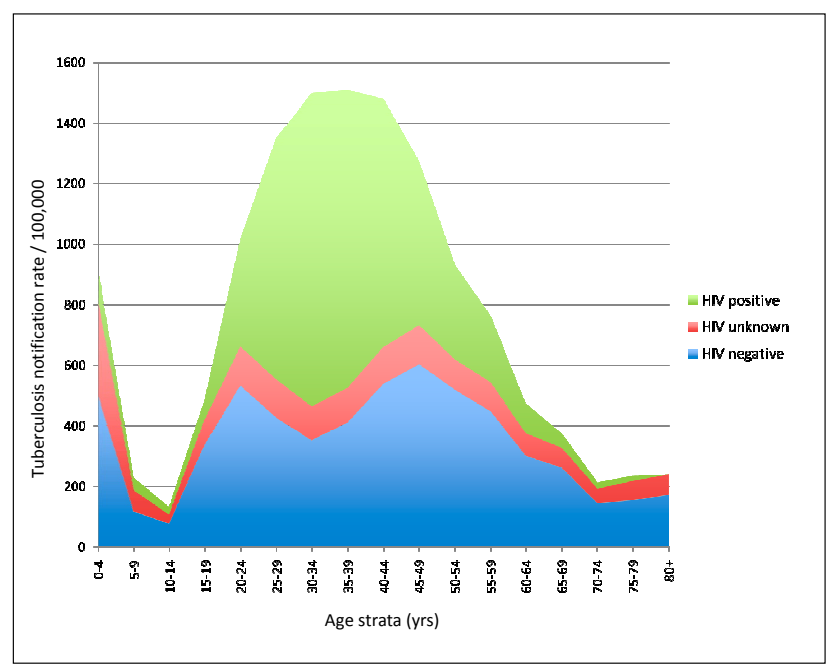

Fig. 1. TB notifications in 2009 for the City of Cape Town stratified by 5-year age groups and by provider-initiated HIV testing results. The demominators for age strata derived from National Department of Health/Health Information System Programme by disaggregating StatsSA district estimates (November 2009) using data from the 'Small Area Layer' (StatsSA, 2004). 
rate was among young adults, exceeding 1400 per 100000 population (Fig. 1). Of those aged between 25 years and 45 years, $63 \%$ of TB cases were HIV-associated. Of particular concern is that about $1 \%$ of children $<5$ years old were notified as having TB. Since children rapidly progress from $\mathrm{TB}$ infection to $\mathrm{TB}$ disease, childhood $\mathrm{TB}$ disease indicates recent ongoing TB transmission. ${ }^{4,5}$ These data strongly indicate very high transmission rates in the community.

The cumulative lifetime risk of new and recurrent TB of HIVuninfected individuals living in Cape Town was calculated by cumulatively adding the annual incidence of each 5 -year age grouping (Fig. 2). The denominators for these calculations are the total age strata populations. As the HIV-infected population is included in the denominator, the resultant rate estimations are conservative. With the status quo, 1 in 5 individuals resident in Cape Town who remain HIV-uninfected will be at risk for developing TB before reaching the age of 60 years. This modern lifetime risk is unprecedented and is approximately twice that for individuals acquiring $\mathrm{TB}$ infection in the UK in the 1950s. ${ }^{6}$ The cumulative risk of TB disease for those who become HIV-infected is considerably higher. Therefore our TB control strategies have failed, resulting in the highest reported global rates of TB disease in children and HIV-infected and uninfected adults.

\section{TB infection}

The epidemiological transitions relevant to the pathogenesis of TB from exposure to death or cure are outlined in Fig. 3. As with other infectious diseases, the key primary event is the initial acquisition of infection. While there have been no recent systematic attempts to measure TB infection rates in South Africa, data from Cape Town report extremely high prevalence rates of TB infection among children and adolescents. ${ }^{7-10}$

In 2005 , the prevalence of a positive ( $>10 \mathrm{~mm}$ induration) tuberculin skin test (TST) among 7457 primary school children (median age 8.6 years) was $37.4 \% .^{7}$ A TST survey in a Cape Town school in 2005 reported TB prevalence to be $26.2 \%$ in 5 - 8 -yearolds, increasing to $52.5 \%$ in 14 - 17 -year-olds. ${ }^{8}$ The prevalence of TB infection increased from $20 \%$ at school entry to $52 \%$ at 15 years, and reached $75 \%$ at 25 years in another study including HIV-negative adolescents and adults. ${ }^{9}$ Note also that a further $8 \%$ of the population will have developed TB disease by the age of 25 years (Fig. 2) and they were excluded from the prevalence surveys. In 2 neighboring urban communities with low HIV prevalence, a high prevalence of TB infection in children (6 - 9 years) was reported. ${ }^{10}$ Transmission rates

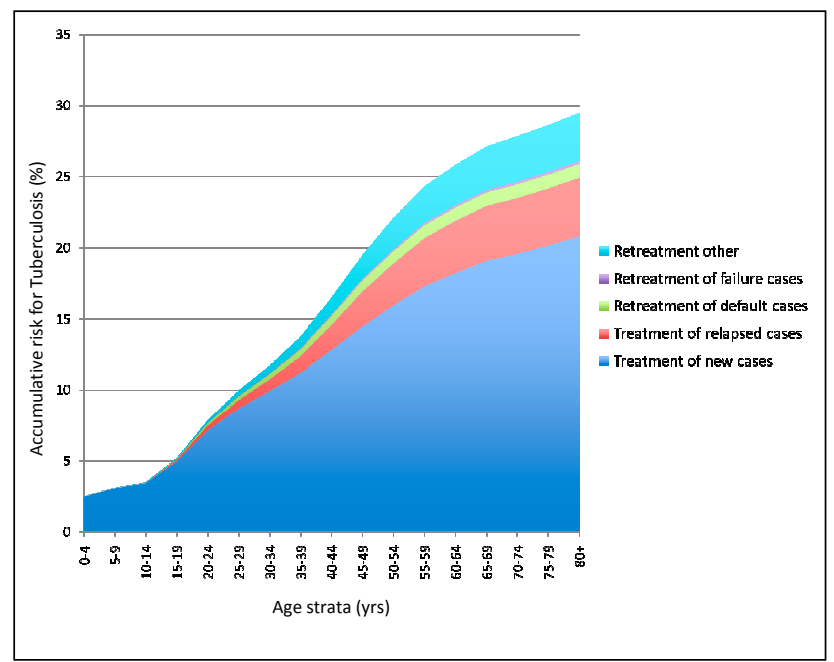

Fig. 2. The accumulated life-time risk of being notified with new or relapsed $T B$ calculated for HIV-negative individuals. Values are based on cumulative 2009 age-specific TB notification rates for Cape Town.

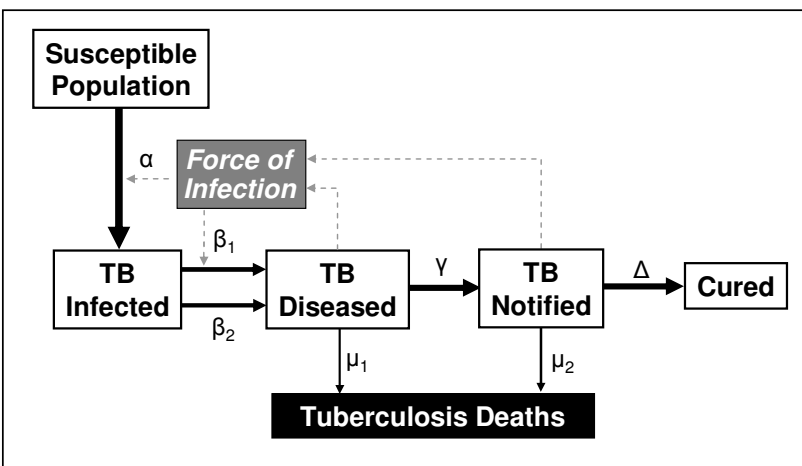

Fig. 3. An outline of TB transition states for a susceptible population from initial infection to disease and treatment outcome or death. The force of infection is shown as a function of prevalent untreated TB disease and determines the rate of primary TB infection ( $\alpha)$ and the rate of multiple infection exposures $\left(\beta_{1}\right)$ of latently infected individuals. $\beta_{2}$ is the progression rate from latent to active disease, $\gamma$ the case-finding proportion, $\Delta$ the cure rate, and $\mu_{1}$ and $\mu_{2}$ the off-and on-chemotherapy mortality rates respectively.

increased between 1998 and 2005 and remained among the highest (4.1 - 5.8\% per annum) in the world. ${ }^{10}$

Collectively, these studies indicate an extremely high prevalence of TB infection in Cape Town acquired during childhood, and that high rates of acquisition of TB infection continue throughout adolescence and into young adulthood. These high rates of TB transmission predate the HIV epidemic and have not declined over the last decade. Together with increasing $\mathrm{TB}$ notification rates, these data clearly indicate a failure of TB control.

\section{Force of TB infection}

The force of infection is the proportion of TB-uninfected individuals newly infected per annum, and is principally determined by the prevalence of infectious $\mathrm{TB}$ cases and the effective number of secondary cases infected by each infectious case. A TB prevalence survey of a random sample of the general population was conducted in a Cape township in 2005. The prevalence of laboratory-proven TB among HIV-uninfected and infected adults was $0.47 \%$ and $5.2 \%$ respectively. ${ }^{11}$ After the community scale-up of antiretroviral therapy (ART), the prevalence of TB among the HIV-uninfected population did not change, but the prevalence among HIV-infected adults decreased from $5.2 \%$ to $1.3 \% .^{12}$

Cape Town studies estimated the force of TB infection to be between $4 \%$ and $8 \%$ per annum. ${ }^{7-10}$ In historical perspective, these values are similar to the force of infection estimated for the UK population in the early decades of the 20th century, which was long before TB chemotherapy was developed. In 1900 in the UK, the force of infection was $10 \%$ per annum, declining to $1 \%$ around 1950 and to $<0.01 \%$ by $2000 .^{13}$

Fig. 4 shows the relationship between increasing force of infection and the proportion of the population with primary TB infection and the proportion multiply exposed to TB infection. Effective TB control interventions that produced small reductions in the force of infection in Cape Town could produce large benefits in transmission reduction. Reduction of the force of infection to $1 \%$ would decrease the proportion of the population exposed to multiple TB exposures from over $50 \%$ to $<0.5 \%$ ( $\beta_{1}$ in Fig. 3 ) and the proportion acquiring primary infection would decrease from $75 \%$ to $<30 \%$ ( $\alpha$ in Fig. 3 ).

\section{Effective contact number}

An effective TB contact is defined as a contact between an infectious pulmonary case and a susceptible individual, sufficient to result in TB infection. ${ }^{14}$ The number of individuals infected by each case (the 


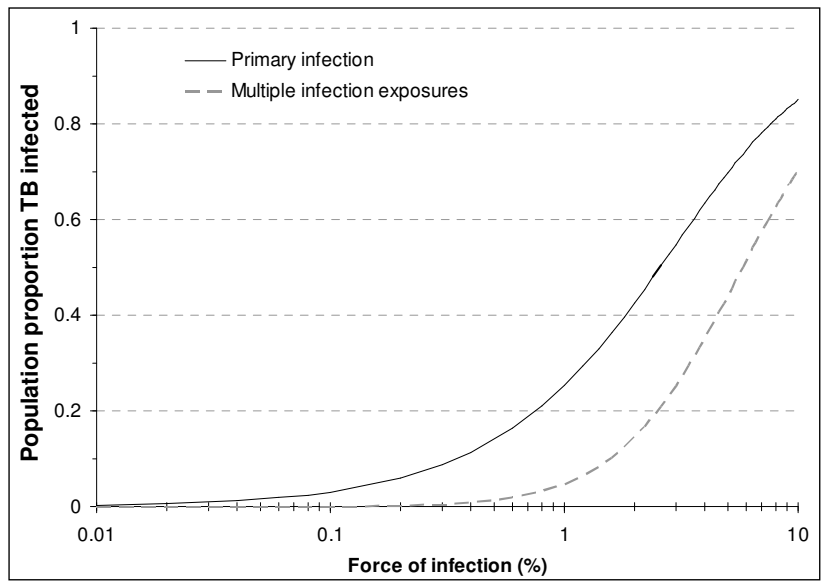

Fig. 4. The relationship between 'force of tuberculosis infection' and proportion of population acquiring primary $T B$ infection and multiple infectious exposures. The prevalence of primary infection is defined as $\mathrm{P}_{1}=\left[{ }^{60} \sum_{\mathrm{x}=0}(1-\exp (-\mathrm{Fx})] / 61\right.$ and the prevalence of multiple infection exposures is defined as $\mathrm{P}_{2}=\left[{ }^{60} \Sigma_{\mathrm{x}=0}(1-\exp (-\right.$ $\mathrm{Fx})-(\mathrm{Fx}) \exp (-\mathrm{Fx})] / 61$ where $F$ is force of TB infection and $x$ is age.

effective contact number) is determined by the ratio of the force of infection and the prevalence of infectious pulmonary TB cases. The ratio between a force of infection of $4-8 \%$ (4 $000-8000 / 100000)$ TB infection rate and a prevalence of smear-positive pulmonary cases notified during 2009 in Cape Town of 370/100 000 indicates an effective contact number of between 11 and 22 . Historically, the effective contact number in the UK declined from 22 in 1900, to 10 in 1950 and to 1 in $1990 .{ }^{13}$

For long-term control of an epidemic, the effective contact number must be lower than the number of individuals who can be expected to produce a single new case of infectious pulmonary TB during a lifetime. As the lifetime risk in Cape Town of developing smear-positive pulmonary TB among HIV-negative residents is approximately $10 \%$, the effective contact number must be reduced to $<10$ to achieve a reduction in the TB epidemic. Current interventions are not achieving this.

\section{Impact of the HIV epidemic}

The South African HIV epidemic has expanded markedly over 20 years, and it was recognised early that $\mathrm{TB}$ was a major cause of morbidity and mortality. ${ }^{15,16} \mathrm{~A}$ pertinent question is the degree to which the HIV-associated TB epidemic has caused the failure to control $\mathrm{TB}$ or the manifestation of the failure to contain $\mathrm{TB}$ transmission. Although $44 \%$ of the total TB case load in Cape Town in 2009 was HIV-related, only $14.3 \%$ of smear-positive pulmonary disease was HIV-related. It therefore appears likely that the HIV epidemic disproportionately increases TB case load rather than TB transmission.

There is no strong evidence that HIV-associated immune suppression affects the acquisition of TB infection ( $\alpha$ Fig. 3). Instead, HIV infection appears to be associated with a marked increase in risk of progression from latent infection to TB disease, following either a primary ( $\beta_{2}$ Fig. 3$)^{17-19}$ or recurrent exposure $\left(\beta_{1} \text { Fig. } 3\right)^{20,21}$ Case-finding proportions ( $\gamma$ Fig. 3 ) were reduced in HIV-infected individuals (44\%) compared with HIV-uninfected (57\%) in a Cape Town township. ${ }^{7}$ However, the population case-finding proportion improved following introduction of an ART programme (64\%). ${ }^{8} \mathrm{~TB}$ case fatality ( $\mu_{1}$ and $\mu_{2}$ Fig. 3 ) is increased particularly in those with low CD4 cell counts. ${ }^{22}$ ART partially reverses immune suppression ${ }^{23}$ and can reduce risk of TB progression after recent TB exposure $\left(\beta_{1}\right.$
Fig. 3) and, together with isoniazid preventive therapy, can decrease the progression from latent to active disease $\left(\beta_{2}\right.$ Fig. 3). ${ }^{24}$ Active case-finding within ART programmes markedly improves TB case finding. ${ }^{12,25-28}$

\section{Why is TB control failing?}

Broad existing strategies for $\mathrm{TB}$ control are case-finding and treatment of active disease, treatment of latent $\mathrm{TB}$ infection, and vaccination with bacille Calmette-Guérin (BCG). ${ }^{25}$ Universal BCG vaccination of all infants protects against progression to miliary $\mathrm{TB}$ and TB meningitis but does not affect TB transmission. ${ }^{29}$ Treatment of latently infected individuals with isoniazid prophylaxis has not been widely implemented in either HIV-infected or uninfected populations in SA. ${ }^{2}$

The SA TB control strategy predominantly focuses on the quality of case management of patients passively presenting to $\mathrm{TB}$ clinics. Passive case-finding is detection of active TB disease among symptomatic patients presenting to medical services, and is promoted in developing countries as part of the WHO-recommended DOTS strategy. ${ }^{30,31}$ Consequently, the primary targets and reporting statistics of the SA TB control programme has been the proportion of TB cases which are effectively treated under DOTS with anti-TB chemotherapy. ${ }^{1,2}$ SA national DOTS coverage increased from $77 \%$ to $100 \%$ and the treatment success rate from $61 \%$ to $74 \%$ between 2001 and 2006. ${ }^{1,2}$ However, TB notifications doubled in the same period. ${ }^{1,2}$

The TB burden was decreasing in industrialised countries before effective chemotherapy was introduced, with reductions in the force of infection from approximately $10 \%$ to $1 \%$ in the early 20 th century. ${ }^{13}$ Introducing effective chemotherapy in the 1950s consolidated these trends in improved TB control. While effective TB case management is necessary in TB control, it was, however, predicted that it would be insufficient for TB control in scenarios such as South Africa with a high force of infection, high proportion of latently infected individuals and a generalised HIV epidemic. ${ }^{32}$ The DOTS strategy is insufficient in high HIV-burdened settings. ${ }^{33}$ In high transmission settings where effective contact numbers are high, lower case-finding rates and delays in diagnosis and initiation of chemotherapy result in ongoing transmission.

\section{Development of a new TB control strategy}

The benefits of improved case-finding depend on the prevailing epidemiology of $\mathrm{TB}$ transmission. In a setting with a force of infection $<1.0$, detecting a case of $\mathrm{TB}$ will mainly benefit that individual alone. In contrast, the benefit of early detection of a TB case where there is an effective contact number $>10$ will additionally prevent up to 10 secondary cases. The benefits of increased and earlier case-finding on TB transmission are therefore significantly amplified in high-transmission settings. Decreasing TB infection rates is fundamental to achieving the long-term aim of TB control of a steady decline of disease in successive generations. Reducing the high force of TB infection, especially in high-density townships, should therefore become a primary target for long-term TB control. Historical TB control measures using community-based interventions such as enhanced and intensified case-finding strategies must be re-explored in view of the additional benefits accruing for decreasing transmission. ${ }^{25,34,35}$

Reducing the time period of infectiousness also directly influences the prevalence of infectious TB. The period of infectiousness results from delays including health-seeking behaviour, diagnostic delays and health systems delays in initiating effective chemotherapy. Intensified 
case-finding can increase awareness of typical symptoms of TB disease, thereby improving health-seeking behaviour. Diagnostic delays can be reduced by using newer molecular diagnostic technologies, and improved health systems efficiencies can further decrease time to initiation of effective TB therapy.

High-risk communities should be specifically targeted, and agespecific interventions are necessary to interrupt $\mathrm{TB}$ transmission to infants and young children, school-age children and adolescents, and both HIV-negative and positive adult populations. A change in priority focus from case management to $\mathrm{TB}$ transmission reduction should be accompanied by incorporating new outcome measures that reflect ongoing TB transmission at national and sentinel sites. A reduction in TB disease rates among young children, and a steady decrease in the number of latently infected children at school entry and subsequently, would reflect a decrease of $\mathrm{TB}$ transmission to children. TB control among adults would be reflected by a decrease in the proportion of recent infections, a decrease in the effective contact number, and eventually a decrease in lifetime risk of TB disease. In HIV-infected patients, full implementation of existing ART guidelines will reduce the pre-ART TB disease burden. The TB infection rates of patients on ART probably reflect the current force of TB infection. The effectiveness of 6-month isoniazid prophylaxis therapy (IPT) to reduce $\mathrm{TB}$ disease in already latently infected individuals is very likely undermined where the force of TB infection is high and consistent with southern African data indicating little or no lasting benefit after 6 months IPT. ${ }^{36,37}$

Changing the emphasis from individual benefit to population benefit has parallels with the concept of using ART as prevention, which has been modelled as a potential strategy to control the HIV epidemic. $^{38,39}$ This may have an additional effect on control of HIVassociated TB. ${ }^{40}$ The HIV force of infection is the result of prevalent community levels of HIV load and sexual networking, which can be reduced by widespread HIV testing and initiation of ART. Similarly for the TB epidemic, the drivers of the high force of infection are population prevalence of infectious $\mathrm{TB}$ cases and the effective contact number. Reducing the high force of TB infection, especially in highdensity townships, should therefore become a primary target for long-term TB control.

RW and LGB were funded in part by the National Institutes of Health (NIH) through CIPRA grant 1U19AI53217-01 and RO1 grant A105873601A1. SDL was funded by the Wellcome Trust, London, UK. The funding sources played no part in the decision to publish these data.

We thank Judy Caldwell, Project Manager, and the City of Cape Town's Health Department TB Control Progamme for providing TB notifications data for the City of Cape Town for 2009, and Calle Hedberg of Health Management Information Systems, City of Cape Town Health Department, for providing the age stratified Cape Town population for 2009. This was estimated by the National Department of Health/ Health Information System Programme by disaggregating StatsSA district estimates (November 2009) using data from the 'Small Area Layer' (StatsSA, 2004)

\section{References}

World Health Organization. Global Tuberculosis Control. A Short Update to the 2009 Report. Report No. WHO/HTM/TB/2009.426. Geneva: World Health Organization, 2009.

2. World Health Organization. Global Tuberculosis Control 2009. Epidemiology, Strategy, Financing Report No: WHO/HTM/TB/2009.411. Geneva: World Health Organization, 2009.
Kaplan R, Bekker L-G, Caldwell J, et al. HIV prevalence, CD4 count distribution and case fatality for TB patients treated in primary health care facilities in Cape Town. Abstracts of the 2nd South African TB patients treated in primary health care facilities in Cape Town. Absto

Reider HL. Epidemiological basis of tuberculosis control. Paris: International Union against Tuberculosis and Lung Disease, 1999.

5. van Rie A, Beyers N, Gie RP, Kunneke M, Zietsman L, Donald PR. Childhood tuberculosis in an urban population in South Africa: burden and risk factor. Arch Dis Child 1999;80:433-437.

Sutherland I. The ten-year incidence of clinical tuberculosis following "conversion" in 2550 individuals aged 14 to 19 years. The Hague, The Netherlands: TSRU Progress Report; KNCV, 1968.

. Shanaubel K, Sismanidis C, Ayles H, et al. Annual risk of tuberculous infection using different methods in communities with a high prevalence of TB and HIV in Zambia and South Africa. PlosOne 2009; 4(11) e 7749 .

8. Middelkoop K, Bekker LG, Myer L, Dawson R, Wood R. Rates of tuberculosis transmission to children and adolescents in a community with a high prevalence of HIV infection among adults. Clin Infect Dis 2008;47:349-355.

9. Wood R, Hua Liang, Lawn SL, Middelkoop K, Wilkinson R, Hulin Wu. Prevalence of latent mycobacterium tuberculosis infection in adolescents and young adults in Cape Town, South Africa. Int J Tuberc Lung Dis 2010;14(4):406-412.

10. Kritzinger FE, Den Boon S, Verver S, et al. No decrease in annual risk of tuberculosis infection in endemic area in Cape Town, South Africa. Trop Med Int Health 2009;14(2):136-142.

11. Wood R, Middelkoop K, Myer L, et al. Undiagnosed tuberculosis in a community with high HIV prevalence: implications for tuberculosis control. Am J Respir Crit Care Med 2007;175:87-93.

12. Middelkoop K, Bekker L-G, Myer L, et al. Antiretroviral program associated with reduction in untreated prevalent tuberculosis in a South African township Am J Respir Crit Care Med 2010; 25 June (epub ahead of print).

13. Vynnycky E, Fine PEM. Interpreting the decline in tuberculosis: the role of secular trends in effective contact. Int J Epidemiol 1999;28:327-334.

14. Abbey H. An examination of the Reed-Frost theory of epidemics. Hum Biol 1952;24:201-233.

15. Bekker L-G, Orrell C, Reader L, et al. Antiretroviral therapy in a community clinic: Early lessons from a pilot project. S Afr Med J 2003;93(6):458-462.

16. Holmes CB, Wood R, Badri M, et al. CD4 decline and incidence of opportunistic infections in Cape Town, South Africa. Implications for prophylaxis and treatment. JAIDS 2006:42(4):464-469.

17. Selwyn PA, Alcabes P, Hartel D, et al. Clinical manifestations and predictors of disease progression in Selwyn PA, Alcabes P, Hartel D, et al. Clinical manifestations and predictors of disease progression
drug users with human immunodeficiency virus infection. N Engl J Med 1992;327(24):1697-1703.

18. Rieder HL, Cauthen GM, Bloch AB, et al Tuberculosis and acquired immunodeficiency syndromeRieder HL, Cauthen GM, Bloch AB, et al Tub
Florida. Arch Intern Med 1989;149:1268-1273.

Florida. Arch Intern Med 1989;149:1268-1273.
9. Center for Disease Control. Tuberculosis and acquired immunodeficiency syndrome-New York City. Morb Mortal Wkly Rep 1987;35:785-796

20. Di Perri G, Cruciani M, Danzi MC, et al. Nosochomial epidemic of active tuberculosis among HIVinfected patients. Lancet 1989;2:1502-1504.

21. EdlinBR, Tokars JI, Grieco HM, et al. An outbreak of multidrug-resistant tuberculosis among hospitalized patients with acquired immunodeficiency syndrome. N Engl J Med 1992;326:1514-1521.

22. Lawn SD, Myer L, Bekker L-G, Wood R. Burden of tuberculosis in a South African antiretroviral treatment (ART) service: impact on ART outcomes and implications for TB control. AIDS 2006;20(12):1605-1612.

23. Lawn SL, Myer L, Edwards D, Bekker L-G, Wood R. Short-term and long-term risk of tuberculosis associated with CD4 cell recovery during antiretroviral therapy in South Africa. AIDS 2009:23(13):17171725.

24. Lawn SD, Wood R, De Cock KM, Kranzer K, Lewis I, Churchyard G. Antiretrovirals and isoniazid preventive therapy in the prevention of HIV-associated tuberculosis in resource-constrained settings. Lancet Infect Dis 2010;10:489-498.

25. Golub JE, Mohan CI, Comstock GW, Chaisson RE. Active case finding of tuberculosis: historical perspective and future prospects Int J Tuberc Lung Dis 9(11):1183-1203.

26. Kranzer K, Houben R, Glynn JR, Bekker LG, Wood R, Lawn SD. Yield of HIV-associated tuberculosis during intensified case finding in resource-limited settings: systematic review and meta-analysis. Lancet Infect Dis 2010;10:93-102.

27. Lawn SD, Kranzer K, Edwards DJ, McNally M, Bekker LG, Wood R. Tuberculosis during the first year of antiretroviral therapy in a South African cohort using an intensive pre-treatment screening strategy. AIDS 2010;24:1323-1328

28. Bassett IV, Wang B, Chetty S, et al. Intensive tuberculosis screening for HIV-infected patients starting antiretroviral therapy in Durban, South Africa. Clin Infect Dis 2010;51(7):823-829.

29. World Health Organization. WHO Position Paper on BCG Vaccination. Wkly Epidemiol Rec 2004;79:25-40.

30. World Health Organization. The Stop TB Strategy: building on enhancing DOTS to meet the TB related Millenium Development Goals. Report No: WHO/HTM/TB/2006.368. Geneva: World Health Organization, 2006.

31. World Health Organization. The Global Plan to Stop TB, 2006-2015: actions for life towards a world free of tuberculosis. Report No: WHO/STB/2006.35. Geneva: World Health Organization, 2006.

32. Styblo K. Impact of HIV infection on the tuberculosis problem worldwide. Kekkaku 1990;65:429-438.

33. De Cock KM, Chaisson RE. Will DOTS do it? A reappraisal of tuberculosis control in countries with high rates of HIV infection. Int J Tuberc Lung Dis 1999;3:457-465.

4. Becerra MC, Pacheo-Torreblanca IF, Boyona J, et al. Expanding tuberculosis case detection by screening household contacts. Public Health Rep 2005;120:271-277.

35. Corbett EL, Bandason T, Duong T, et al. Comparison of two active case-finding strategies for community-based diagnosis of symptomatic smear-positive tuberculosis and control of infectious tuberculosis in Harare, Zimbabwe (DETECTB): a cluster-randomised trial. Lancet 2010; (epub ahead of print).

36. Samandari T. Preliminary results of the Botswana isoniazid preventive therapy (IPT) clinical trial. 40th Union World Conference on Lung Health. Cancun, Mexico: Dec. 3 - 72009.

37. Golub JE, Pronyk P, Mohapi L, et al. Isoniazid preventive therapy, HAART and tuberculosis risk in HIVinfected adults in South Africa: a prospective cohort. AIDS 2009;23(5):631-666.

38. Montaner JSG, Hogg R, Wood E, et al. The case for expanding access to highly active antiretroviral Montaner JSG, Hogg R, Wood E, et al. The case for expanding access to
therapy to curb the growth of the HIV epidemic. Lancet 2006;368:531-536.

39. Granich RM, Gilks CF, Dye C, De Cock KM, Williams BG. Universal voluntary HIV testing with immediate antiretroviral therapy as a strategy for elimination of HIV transmission: a mathematical model. Lancet 2009;373:48-57.

40. Lawn SD, Anthony D, Harries AD, et al. Will ART do it? Antiretroviral therapy for control of HIVassociated tuberculosis. Int J Tuberc Lung Dis 2010 (in press).

Accepted 28 October 2010 